\title{
Erratum to: Investigation of the effect of precoalescence or postcoalescence crosslinking on film formation, properties, and latex morphology
}

Ravi G. Joshi, Theodore Provder, Paul Ziemer, Wenjing Mao, Weidian Shen, Frank N. Jones

Erratum to: J. Coat. Technol. Res. (2009) 6(1): 47-65

\section{DOI 10.1007/s11998-008-9115-7}

The results reported in Table 5: Results of stress-strain analysis were not calculated correctly. The correct values for Table 5 are given.

In addition, the text on the previous page, column 1, needs modification. It was "However, a significant decrease in the area under the curve and strain at break is observed. The sample becomes less flexible. Going from $0 \%$ to $1 \%$ crosslinker for the low $T_{\mathrm{g}}$ latex shows hardly any difference in Young's modulus. However from $1 \%$ to $5 \%$ of crosslinker Young's modulus values go from approximately 20 to $38 \mathrm{MPa}$."

It should be modified to "However, the area under the curve goes through a maximum at $1 \%$ and then levels out to a lower value at $5 \%$, while a decrease in the strain at break is observed. The sample becomes less flexible. Going from $0 \%$ to $1 \%$ crosslinker for the low $T_{\mathrm{g}}$ latex shows an increase in Young's modulus from 9 to $20 \mathrm{MPa}$. However, from $1 \%$ to $5 \%$ of crosslinker Young's modulus values go from approximately 20 to $45 \mathrm{MPa}$."

The online version of the original article can be found under doi:10.1007/s11998-008-9115-7.

R. G. Joshi, P. Ziemer, W. Mao, W. Shen,

F. N. Jones

Coatings Research Institute, Eastern Michigan University,

Ypsilanti, MI 48197, USA

T. Provder $(\square)$

Polymer \& Coatings Consultants, LLC, 5645A Emerald

Ridge Pkwy., Solon, OH 44139, USA

e-mail: tprovder@att.net 
Table 5: Results of stress-strain analysis

\begin{tabular}{|c|c|c|c|c|c|}
\hline Sample & $\begin{array}{c}\text { Gel } \\
\text { content }\end{array}$ & $\begin{array}{l}\text { Young's modulus } \\
\left(E^{\prime}\right)(\mathrm{MPa})\end{array}$ & Area under curve & $\begin{array}{c}\text { Strain } \\
\text { at break }\left(\varepsilon_{\mathrm{b}}\right)(\%)\end{array}$ & $\begin{array}{c}\text { Stress } \\
\text { at break }\left(\varepsilon_{\mathrm{b}}\right)(\%)\end{array}$ \\
\hline IL-0 & 0.00 & $8.9 \pm 0.6$ & $172 \pm 11$ & $95.7 \pm 6$ & $2.7 \pm 0.9$ \\
\hline IL-0.25 & 46.1 & $13.9 \pm 0.9$ & $261 \pm 21$ & $109 \pm 5.4$ & $5.35 \pm 0.6$ \\
\hline IL-0.6 & 56.2 & $16.7 \pm 1.2$ & $299 \pm 12$ & $106 \pm 5.4$ & $5.78 \pm 0.5$ \\
\hline IL-1.2 & 52.8 & $16.2 \pm 2.7$ & $271 \pm 40$ & $107 \pm 15$ & $5.65 \pm 0.8$ \\
\hline IL-2 & 57.1 & $18.1 \pm 0.9$ & $311 \pm 14$ & $106 \pm 12$ & $6.00 \pm 0.2$ \\
\hline IL-4 & 64.2 & $49.7 \pm 9.2$ & $222 \pm 6.3$ & $57.9 \pm 1.2$ & 7.7 \\
\hline $\mathrm{IH}-\mathrm{O}$ & 0.0 & $15.9 \pm 1.1$ & $175 \pm 2.8$ & $98.8 \pm 0.1$ & 3.55 \\
\hline $\mathrm{IH}-0.25$ & 10.1 & $55.5 \pm 4.6$ & $415 \pm 68$ & $74.7 \pm 9.6$ & $8.66 \pm 0.6$ \\
\hline $\mathrm{IH}-0.6$ & 10.3 & $55.5 \pm 4.6$ & $415 \pm 68$ & $74.7 \pm 9.6$ & $8.66 \pm 0.6$ \\
\hline $\mathrm{IH}-1.2$ & 15.6 & $59.9 \pm 1.3$ & $459 \pm 25$ & $75.5 \pm 4.6$ & $9.67 \pm 0.3$ \\
\hline $\mathrm{IH}-2$ & 61.3 & $15.2 \pm 1.6$ & $257 \pm 28$ & $105 \pm 11$ & $5.04 \pm 0.4$ \\
\hline $\mathrm{IH}-4$ & 62.9 & $213 \pm 5.7$ & 186 & 38.2 & 9.83 \\
\hline EL-O & 0.0 & $8.9 \pm 0.6$ & $172 \pm 11$ & $95.7 \pm 6$ & $2.7 \pm 0.9$ \\
\hline EL-1 & 91.3 & $19.5 \pm 0.5$ & $318 \pm 57$ & $93.2 \pm 11$ & $6.71 \pm 0.6$ \\
\hline EL-2 & 98.1 & $24.2 \pm 1.5$ & $221 \pm 50$ & $62.9 \pm 9.1$ & $6.63 \pm 0.8$ \\
\hline EL-5 & 100.0 & 45.3 & $196.5 \pm 11$ & $41.8 \pm 0.7$ & $9.5 \pm 0.5$ \\
\hline $\mathrm{EH}-\mathrm{O}$ & 0.0 & $15.9 \pm 1.1$ & $175 \pm 2.8$ & $98.8 \pm 0.1$ & 3.55 \\
\hline $\mathrm{EH}-1$ & 90.0 & $68.9 \pm 10$ & $364 \pm 66$ & $61.8 \pm 7.6$ & $8.71 \pm 0.8$ \\
\hline $\mathrm{EH}-2$ & 97.4 & $69.9 \pm 4.1$ & $361 \pm 35$ & $60.8 \pm 4.1$ & $8.84 \pm 0.3$ \\
\hline $\mathrm{EH}-5$ & 97.9 & $159 \pm 11$ & $174 \pm 11$ & $38.8 \pm 1.5$ & $8.9 \pm 0.2$ \\
\hline
\end{tabular}

